MATHEMATICS OF COMPUTATION

Volume 70, Number 234, Pages 845-851

S $0025-5718(00) 01232-1$

Article electronically published on July 13, 2000

\title{
TENTH DEGREE NUMBER FIELDS WITH QUINTIC FIELDS HAVING ONE REAL PLACE
}

\author{
SCHEHRAZAD SELMANE
}

\begin{abstract}
In this paper, we enumerate all number fields of degree 10 of discriminant smaller than $10^{11}$ in absolute value containing a quintic field having one real place. For each one of the 21509 (resp. 18167) found fields of signature $(0,5)$ (resp. $(2,4))$ the field discriminant, the quintic field discriminant, a polynomial defining the relative quadratic extension, the corresponding relative discriminant, the corresponding polynomial over $\mathbb{Q}$, and the Galois group of the Galois closure are given.

In a supplementary section, we give the first coincidence of discriminant of 19 (resp. 20) nonisomorphic fields of signature $(0,5)$ (resp. $(2,4)$ ).
\end{abstract}

\section{INTRODUCTION}

In this paper, we enumerate all number fields of degree 10 of discriminant smaller than $10^{11}$ in absolute value containing a quintic field having one real place.

The list corresponding to the signature $(0,5)$ (resp. $(2,4)$ ) contains 21509 (resp. 18167) fields. For each field in the given two lists, the field discriminant, the quintic field discriminant, a polynomial defining the relative quadratic extension, the corresponding relative discriminant, the corresponding polynomial over $\mathbb{Q}$, and the Galois group of the Galois closure are given.

To establish these lists, we have followed, without major modification, the method of [4, 7]; Section 2 of this paper contains a brief description of the method used. We describe the results in the third section. The existence of several nonisomorphic fields, of composite fields, of unramified extensions, of Hilbert class fields as well as other characteristics, have been examined in more detail.

Finally, in a supplementary section, we give the first coincidence of a discriminant of 19 (resp. $(2,4))$ nonisomorphic fields of signature $(0,5)$ (resp. $(2,4))$ with a value of its discriminants out of the originally chosen bound.

\section{THE METHOD}

If $L$ is a number field of degree $n$, we denote by $\vartheta_{L}$ its ring of integers, by $d_{L}$ its discriminant, and by $h_{L}$ its class number. For $\beta \in L$, we denote the corresponding conjugates by $\beta^{(1)}, \ldots, \beta^{(n)}$ and set $T_{2}(\beta)=\sum_{i=1}^{n}\left|\beta^{(i)}\right|^{2}$.

To establish the lists of all number fields of degree 10 over $\mathbb{Q}$ and of field discriminant smaller than $10^{11}$ in absolute value containing a quintic subfield having one real place, we have followed without major modification the method of explicit

Received by the editor November 3, 1998 and, in revised form, April 27, 1999.

2000 Mathematics Subject Classification. Primary 11R11, 11R29, 11Y40.

Key words and phrases. Quintic fields, relative quadratic extensions, discriminant.

(C)2000 American Mathematical Society 
construction of quadratic relative extensions as described in [4, 7]. In the following, we are going to briefly describe the main stages that led us to establish the lists for the desired fields.

Let $K$ be a number field of degree $[K: \mathbb{Q}]=10$, of signature $(r, s)$ and of discriminant $d_{K}$ such that $\left|d_{K}\right| \leq 10^{11}$ containing a quintic field $F$ of signature $(1,2)$. According to 4, $K$ contains an integer $\theta, \theta \notin F$ such that

$$
\begin{gathered}
K=F(\theta), \\
\operatorname{Min}(\theta, F)=P(x)=x^{2}+a x+b \in \vartheta_{F}[x], \\
\sum_{i=1}^{10}\left|\theta^{(i)}\right|^{2} \leq \frac{1}{2} \sum_{i=1}^{5}\left|a^{(i)}\right|^{2}+\left(\frac{\left|d_{K}\right|}{4 d_{F}}\right)^{\frac{1}{5}}, \\
r=0 \text { or } 2
\end{gathered}
$$

(since the signature of $F$ must be compatible with that of $K$ ).

To compute all the polynomials $P(x)$ having a root $\theta$ subject to inequality (1), we will work in the field $F$. As

$$
d_{K}=(-1)^{s} d_{F}^{2} N(\delta),
$$

where $\delta$ is the relative discriminant of $K / F$ and $N$ is the absolute norm in the extension $F / \mathbb{Q}$, we must consider all quintic fields $F$ of signature $(1,2)$ with discriminant smaller than $10^{\frac{11}{2}}$. All quintic fields $F$ used were taken from the tables of F. Diaz y Diaz, M. Pohst and A. Schwartz [3].

We denote by $W=\left\{w_{1}=1, w_{2}, \ldots, w_{5}\right\}$ an integral basis of $F$ and by $\sigma_{i}$ $(1 \leq i \leq 5)$ the distinct $\mathbb{Q}$-isomorphisms of $F$ into $\mathbb{C}$, and we set $M=\left(\left|d_{K}\right| / 4 d_{F}\right)^{\frac{1}{5}}$.

According to [4], the coefficient $a$ of $P$ can be chosen in $\vartheta_{F}\left(\bmod 2 \vartheta_{F}\right)$. Moreover, according to 7, each one of the 32 possible values of $a$ can be chosen so that $C=\frac{1}{2} \sum_{i=1}^{5}\left|a^{(i)}\right|^{2}+M$ is minimum.

Once a convenient value of $a$ is determined, we compute the set of suitable values of $b=\sum_{i=1}^{5} b_{i} w_{i}$ from the second relative symmetric function

$$
s_{2}=\theta^{2}+\theta^{\prime 2}=a^{2}-2 b,
$$

where $\theta^{\prime}$ denotes the other root of $P$. The values of $s_{2}$ are computed from the inequality

$$
\sum_{i=1}^{5}\left|s_{2}^{(i)}\right|^{2} \leq C^{2},
$$

which comes from inequality (1) and the inequality

$$
\sum_{i=1}^{5}\left|s_{2}^{(i)}\right| \leq C .
$$

We find ourselves in the presence of a long list of polynomials $P$. The main simplifications used to reduce, as much as possible, the number of polynomials to be considered to construct the complete lists of the desired fields are described below.

First of all, we solved the problem of the signature of the field $K$ by ensuring that the polynomial discriminant $\Delta=a^{2}-4 b$ of the real conjugate polynomial has 
the sign $(-1)^{s}$. However, for the signature $(0,5)$, we also needed to verify that the roots of the other conjugate polynomials are not real for the 50 quintic fields having discriminant square. Then, we proceed with the elimination of polynomials having too large values of $T_{2}(\theta)$ by checking whether the inequality

$$
\sum_{i=1}^{5}\left|\Delta^{(i)}\right| \leq 2 M
$$

is fulfilled. Indeed, since

$$
\sum_{i=1}^{10}\left|\theta^{(i)}\right|^{2}=\frac{1}{2}\left(\sum_{i=1}^{5}\left|a^{(i)}\right|^{2}+\sum_{i=1}^{5}\left|\Delta^{(i)}\right|\right),
$$

then the inequality

$$
\sum_{i=1}^{10}\left|\theta^{(i)}\right|^{2} \leq \frac{1}{2} \sum_{i=1}^{5}\left|a^{(i)}\right|^{2}+M
$$

is equivalent to inequality (2). Finally, we checked the irreducibility of the polynomial $P$ for the signature $(2,4)$.

For the polynomials surviving these tests, we started by computing the relative discriminant $\delta$ using a theorem on ramification in Kummer extensions. Only those polynomials for which $N(\delta) \leq 10^{11} d_{F}^{-2}$ were kept, which allowed us to obtain the value $d_{K}$ directly. As we obtained several polynomials for a given discriminant, we used the function OrderIsSubfield in [1] to decide whether or not such polynomials define the same field up to isomorphism. Then, we computed the polynomial

$$
f(x)=\prod_{i=1}^{5}\left(x^{2}+\sigma_{i}(a) x+\sigma_{i}(b)\right)=\sum_{i=0}^{10} t_{i} x^{10-i} \quad\left(t_{0}=1\right)
$$

and the Galois group of the Galois closure for each field in the lists using KANT [1].

\section{Description of RESUlts, AND Discussion}

In this section, we give a brief discussion of some information provided by these computations.

In Table 1, we give the number of fields that were constructed and we present the number of discriminants for which there are exactly $k$ nonisomorphic fields having the same discriminants.

- The smallest discriminant in the list corresponding to the signature $(0,5)$ is -209352647 with

$$
f(x)=x^{10}-3 x^{9}+8 x^{8}-14 x^{7}+20 x^{6}-22 x^{5}+19 x^{4}-13 x^{3}+6 x^{2}-2 x+1
$$

and

$$
P(x)=x^{2}+\left(-\rho^{4}+2 \rho^{3}-\rho^{2}+\rho-1\right) x+\left(\rho^{4}-\rho^{3}\right) \in \vartheta_{F}[x],
$$

where $\rho$ is a root of

$$
g(x)=x^{5}-2 x^{4}+x^{3}-1 \in \mathbb{Z}[x] .
$$

The relative discriminant being the prime ideal $(23, \rho+19)$ of $F$ over 23. 
TABLE 1.

\begin{tabular}{|c|c|c|}
\hline$(r, s)$ & $(0,5)$ & $(2,4)$ \\
\hline Number of fields & 21509 & 18167 \\
\hline$k$ & \multicolumn{2}{|c|}{ Number of $k$ nonisomorphic fields } \\
\hline 2 & 1996 & 1618 \\
3 & 183 & 176 \\
4 & 131 & 76 \\
5 & 11 & 10 \\
6 & 3 & 1 \\
8 & 5 & 5 \\
9 & 0 & 1 \\
12 & 1 & 1 \\
$>12$ & 0 & 0 \\
\hline
\end{tabular}

TABLE 2 .

\begin{tabular}{|c|c|c|c|c|c|c|c|c|}
\hline \multicolumn{10}{|c|}{$(0,5)$} \\
\hline$d_{L}$ & -3 & -4 & -7 & -8 & -11 & -15 & -19 & -51 \\
\hline$n b$ & 586 & 315 & 29 & 30 & 10 & 2 & 3 & 1 \\
\hline$n b_{1}$ & 162 & 56 & 5 & 2 & - & - & - & - \\
\hline \multicolumn{10}{|c|}{$(2,4)$} \\
\hline$d_{L}$ & 5 & 8 & 13 & 17 & 53 & \multicolumn{3}{|c|}{141} \\
\hline$n b$ & 128 & 23 & 5 & 3 & 1 & \multicolumn{3}{|c|}{1} \\
\hline$n b_{1}$ & 30 & 2 & - & - & - & \multicolumn{3}{|c|}{-} \\
\hline
\end{tabular}

- The smallest discriminant in the list corresponding to the signature $(2,4)$ is 799905449 with

$$
f(x)=x^{10}-x^{9}+2 x^{8}-5 x^{6}-x^{5}+3 x^{4}+4 x^{3}+x^{2}-2 x-1
$$

and

$$
P(x)=x^{2}+\left(-\rho^{2}+2 \rho-1\right) x+\left(-\rho^{3}+2 \rho^{2}-2 \rho+1\right) \in \vartheta_{F}[x],
$$

where $\rho$ is a root of

$$
g(x)=x^{5}-2 x^{4}+2 x^{3}-x^{2}-1 \in \mathbb{Z}[x] .
$$

The relative discriminant $\delta$ being the prime ideal $(41, \rho+4)$ of $F$ over 41 . We notice that there exist no extensions $K / F$ with $\delta=(41, \rho+7)$ and $\delta=$ $(41, \rho+15)$.

Among the fields that we found there were exactly 983 (resp. 161) fields $K$ of signature $(0,5)$ (resp. $(2,4))$ containing, in addition of a quintic field, a quadratic subfield. In Table 2 , we present the number $n b$ of fields $K$ containing the quadratic field $L$ and the number $n b_{1}$ of composite fields $K(K=F . L$ and $F \cap L=\mathbb{Q})$. We have $n b=1$ for 7 fields cited in Proposition 3.2.

Proposition 3.1. Let $F$ be a quintic field of signature $(1,2)$ and of discriminant smaller than 9829 in which 2 remains inert. Let $p \vartheta_{F}=\prod_{i=1}^{t} \wp_{i}^{e_{i}}$ be the decomposition of a prime number $p$ in the field $F$ with $N\left(\wp_{i}\right)=p^{f_{i}}$.

i) There are exactly 4 (resp. 3) nonisomorphic number fields $K$ of signature $(0,5)$ (resp. $(2,4))$ and of discriminant $\left|d_{K}\right|=2^{10} d_{F}^{2}$. 
TABLE 3.

\begin{tabular}{|l|l|l|l|}
\hline$d_{K}$ & \multicolumn{1}{|c|}{$\left(t_{0}, \ldots, t_{10}\right)$} & \multicolumn{1}{|c|}{$\delta$} & $h_{K}$ \\
\hline-229345007 & $(1,-1,6,-3,11,-3,11,-3,6,-1,1)$ & $(47, \rho+17)$ & 1 \\
-3077056399 & $(1,5,18,42,82,120,127,93,42,10,1)$ & $(79, \rho+7)$ & 1 \\
-11592740743 & $(1,4,3,-6,13,93,157,114,36,5,1)$ & $(103, \rho+58)$ & 1 \\
-23863536599 & $(1,3,10,23,45,73,87,65,28,7,1)$ & $(7, \rho+4)(17, \rho+5)$ & 1 \\
- & $(1,2,-4,-10,33,-43,33,-10,-4,2,1)$ & $(7, \rho+3)(17, \rho+15)$ & 2 \\
-33038369407 & $(1,-2,3,1,-3,10,27,-59,41,-3,9)$ & $(127, \rho+80)$ & 1 \\
-38579489651 & $(1,-1,9,4,26,30,37,47,37,18,4)$ & $(131, \rho+104)$ & 1 \\
-59797108943 & $(1,2,6,10,11,10,12,6,4,2,1)$ & $(11, \rho+7)(13, \rho+10)$ & 1 \\
- & $(1,4,9,30,107,253,397,418,292,125,25)$ & $(11, \rho+8)(13, \rho+9)$ & 2 \\
\hline
\end{tabular}

ii) For each $\wp_{i}$ such that $2^{10} p^{f_{i}} d_{F}^{2} \leq 10^{11}$, there exists exactly 4 nonisomorphic fields $K$ of signature $(0,5)$ (resp. $(2,4))$ and of discriminant $\left|d_{K}\right|=2{ }^{10} p^{f_{i}} d_{F}^{2}$.

Remark 3.1. The polynomials $f$ defining the fields $K$ in Proposition 3.1 are even and, moreover, the $f\left(x^{\frac{1}{2}}\right)$ 's are just polynomials defining the corresponding quintic subfield.

Proposition 3.2. Let $F$ be a quintic field of signature $(1,2)$ and of discriminant $d_{F}$ such that $d_{F}=m^{2}$ with $m \leq 10^{\frac{11}{5}}$. Let $L=\mathbb{Q}(\sqrt{-m})$ be the corresponding imaginary quadratic field. For $m \neq 136$, we have $h_{L}=5$.l and there exists $l$ nonisomorphic totally imaginary number fields $K$ containing $F$ and $L$ with $d_{K}=$ $-m^{5}$.

Remark 3.2. The quoted quintic fields in Proposition 3.2 have $D_{5}$ for Galois group.

Remark 3.3. Each field $K$ in Table 3 is a subfield of the Hilbert class field $\mathcal{H}$ of the imaginary quadratic field $L=\mathbb{Q}(\sqrt{-m})$. In particular, for $l=1$ we have $K \simeq \mathcal{H}$.

For $m=136$, we have $h_{L}=4$ and there exists, up to isomorphism, one totally imaginary number field $K$ of discriminant -46525874176 with $K=F(\theta)$ and $F=\mathbb{Q}(\rho)$, where $\theta$ is a root of

$$
p(x)=x^{2}+\left(1-\rho^{2}\right) x+\left(-1+2 \rho-\rho^{2}-\rho^{3}+\rho^{4}\right)
$$

and where $\rho$ is a root of

$$
g(x)=x^{5}-x^{4}+2 x^{2}-x+2 .
$$

The Hilbert class field of the imaginary quadratic field $\mathbb{Q}(\sqrt{-136})$ is defined by the polynomial $x^{8}+12 x^{6}+49 x^{4}+78 x^{2}+4$ of field discriminant 342102016 which contains the quartic subfield of signature $(2,1)$ and of discriminant -2312 .

Proposition 3.3. For each quintic field of signature $(1,2)$ and of discriminant smaller than $10^{11 / 2}$ having class number 2 there exists an unramified extension $K / F$ of signature $(2,4)$.

\section{Supplement}

It is well known that the set of isomorphism classes of number fields of given discriminant is finite (Hermite). With the chosen bound $\Im=10^{11}$ we have obtained up to 12 nonisomorphic fields of discriminant -93771920384 (resp. 93771920384) for the signature $(0,5)$ (resp. $(2,4))$. To our knowledge, for a fixed degree and 
signature, the maximum number of nonisomorphic fields with same discriminant is not known.

Here, we give the first coincidence of 19 nonisomorphic fields for the signature $(0,5)$ and the first coincidence of 20 nonisomorphic fields for the signature $(2,4)$. We think that these numbers are maximum for these kind of fields. In the following, we will describe how we have obtained such results.

Having noticed that the obtained 4, 8 and 12 nonisomorphic fields had a discriminant of the form

$$
\left|d_{K}\right|=2^{10} p^{f_{i}} d_{F}^{2},
$$

where 2 remains inert in the quintic field $F$ and $p$ is a prime number such that $p \vartheta_{F}=\prod_{i=1}^{t} \wp_{i}^{e_{i}}$ with $N\left(\wp_{i}\right)=p^{f_{i}}$, we looked for the first prime number which splits completely in a quintic field where 2 remains inert and such that $2^{10} p d_{F}^{2}$ is as small as possible.

The smallest prime number found, which splits completely, is 83 in the quintic field $F$ of discriminant 2209. Since the target discriminant must have $\left|d_{K}\right|=$ $2^{10} \cdot 83 \cdot 2209^{2}$, we have fixed the bound to $\Im=5 \cdot 10^{11}$. Table 4 contains the results.

We denote by $f_{1}, \ldots, f_{20}$ (resp. $f_{1}^{\prime}, \ldots, f_{19}^{\prime}$ ) the polynomials of signature $(2,4)$ (resp. $(0,5))$ in the order in which they appear in Table 4 . If we denote by

$$
f_{i}(x)=x^{10}+t_{2} x^{8}+t_{4} x^{6}+t_{6} x^{4}+t_{8} x^{2}+t_{10},
$$

then

$$
f \prime_{i}(x)=x^{10}-t_{2} x^{8}+t_{4} x^{6}-t_{6} x^{4}+t_{8} x^{2}-t_{10} .
$$

TABLE 4.

\begin{tabular}{|l|l|}
\hline \multicolumn{1}{|c|}{$(2,4)$} & \multicolumn{1}{c|}{$(0,5)$} \\
\hline$x^{10}+3 x^{8}-5 x^{6}+2 x^{4}+51 x^{2}-83$ & $x^{10}-3 x^{8}-5 x^{6}-2 x^{4}+51 x^{2}+83$ \\
$x^{10}-5 x^{8}+7 x^{6}-16 x^{4}+67 x^{2}-83$ & $x^{10}+5 x^{8}+7 x^{6}+16 x^{4}+67 x^{2}+83$ \\
$x^{10}-8 x^{8}+27 x^{6}-53 x^{4}+85 x^{2}-83$ & $x^{10}+8 x^{8}+27 x^{6}+53 x^{4}+85 x^{2}+83$ \\
$x^{10}-x^{8}-13 x^{6}+17 x^{4}+54 x^{2}-83$ & $x^{10}+x^{8}-13 x^{6}-17 x^{4}+54 x^{2}+83$ \\
$x^{10}-5 x^{8}+12 x^{6}-47 x^{4}+49 x^{2}-83$ & $x^{10}+5 x^{8}+12 x^{6}+47 x^{4}+49 x^{2}+83$ \\
$x^{10}-6 x^{8}+8 x^{6}+5 x^{4}+32 x^{2}-83$ & $x^{10}+6 x^{8}+8 x^{6}-5 x^{4}+32 x^{2}+83$ \\
$x^{10}+x^{6}-28 x^{4}-6 x^{2}-83$ & $x^{10}+x^{6}+28 x^{4}-6 x^{2}+83$ \\
$x^{10}-4 x^{8}-x^{6}-2 x^{4}+24 x^{2}-83$ & $x^{10}-x^{8}-13 x^{6}+48 x^{4}-93 x^{2}+83$ \\
$x^{10}+x^{8}-13 x^{6}-48 x^{4}-93 x^{2}-83$ & $x^{10}+4 x^{8}-x^{6}-18 x^{4}+14 x^{2}+83$ \\
$x^{10}-4 x^{8}-x^{6}+18 x^{4}+14 x^{2}-83$ & $x^{10}+3 x^{8}+16 x^{6}+33 x^{4}+61 x^{2}+83$ \\
$x^{10}-3 x^{8}+16 x^{6}-33 x^{4}+61 x^{2}-83$ & $x^{10}+4 x^{8}-x^{6}+2 x^{4}+24 x^{2}+83$ \\
$x^{10}+5 x^{8}+6 x^{6}-18 x^{4}-71 x^{2}-83$ & $x^{10}-5 x^{8}+6 x^{6}+18 x^{4}-71 x^{2}+83$ \\
$x^{10}-5 x^{8}+x^{6}+14 x^{4}+31 x^{2}-83$ & $x^{10}+5 x^{8}+x^{6}-14 x^{4}+31 x^{2}+83$ \\
$x^{10}+10 x^{8}+32 x^{6}+31 x^{4}-20 x^{2}-83$ & $x^{10}-10 x^{8}+32 x^{6}-31 x^{4}-20 x^{2}+83$ \\
$x^{10}+4 x^{8}-x^{6}-31 x^{4}-81 x^{2}-83$ & $x^{10}-4 x^{8}-x^{6}+31 x^{4}-81 x^{2}+83$ \\
$x^{10}+5 x^{8}+18 x^{6}+15 x^{4}+x^{2}-83$ & $x^{10}-5 x^{8}+18 x^{6}-15 x^{4}+x^{2}+83$ \\
$x^{10}+x^{8}+9 x^{6}+19 x^{4}-42 x^{2}-83$ & $x^{10}-x^{8}+9 x^{6}-19 x^{4}-42 x^{2}+83$ \\
$x^{10}-8 x^{8}+19 x^{6}-17 x^{4}+41 x^{2}-83$ & $x^{10}+8 x^{8}+19 x^{6}+17 x^{4}+41 x^{2}+83$ \\
$x^{10}-7 x^{8}-33 x^{4}+x^{2}-83$ & $x^{10}+7 x^{8}+33 x^{4}+x^{2}+83$ \\
$x^{10}+8 x^{6}-19 x^{4}+10 x^{2}-83$ & \\
\hline
\end{tabular}


Notice that the polynomial $f \prime_{20}(x)=x^{10}+8 x^{6}+19 x^{4}+10 x^{2}+83$ defines a totally imaginary number field of degree 10 of discriminant $-83 \cdot 2209^{2}$. Let $y=x^{\frac{1}{2}}$; we have $f \prime_{i}(y)=-f_{i}(-y)$, and moreover $f_{i}(y)$ and $f \prime_{i}(y)$ are just polynomials defining the quintic subfield $F$.

All computations have been done on a Pentium MMX 200.

\section{ACKNOWLEDGMENT}

I thank Professor F. Diaz y Diaz.

\section{REFERENCES}

[1] M. Daberkow, C. Fieker, J. Klüners, M. Pohst, K. Roegner and K. Wildanger, Kant V4, J. Symbolic Comp. 24 (1997), 267-283. MR 99g:11150

[2] F. Diaz y Diaz, Private communication to the author.

[3] F. Diaz y Diaz, M. Pohst and A. Schwartz, A table of quintic number fields of signature $(1,2)$, Math. Comp. 56 (1991), 801-808. MR 91h:11155

[4] J. Martinet, Methodes geometriques dans la recherche des petits discriminants, Sem. de Theorie des nombres de paris 1983/1984, Birkhauser Verlag, Bassel (1985) 147-179. MR 88h:11083

[5] M. Pohst, On computing isomorphisms of equation orders, Math. Comp. 48 (1987), 309-314. MR 88b:11066

[6] Sc. Selmane, Nonprimitive number fields of degree eight and of signature $(2,3),(4,2)$ and $(6,1)$ with small discriminant, Math. Comp. 68 (1999), 333-344. [MR 99c:11160]

[7] Sc. Selmane, Quadratic extensions of totally real quintic fields, Math. Comp. (this journal, previous article). CMP 99:15

University of Sciences and Technology (U.S.T.H.B), Institut of Mathematics, B.P. 32 El Alia, Bab-Ezzouar, 16111, Algiers, Algeria

E-mail address: sc_selmane@hotmail.com 